21st Particles and Nuclei International Conference (PANIC 2017)

International Journal of Modern Physics: Conference Series

Vol. 46 (2018) 1860072 (6 pages)

(C) The Author(s)

DOI: $10.1142 / \mathrm{S} 2010194518600728$

\title{
Studies of Missing Energy Decays at Belle II
}

\author{
Yinghui Guan (on Behalf of the Belle II Collaboration) \\ High Energy Accelerator Research Organization, KEK, \\ Tsukuba, 305-0801, Japan \\ guanyh@post.kek.jp
}

Published 3 May 2018

\begin{abstract}
The Belle II experiment at the SuperKEKB collider is a major upgrade of the KEK "B factory" facility in Tsukuba, Japan. The machine is designed for an instantaneous luminosity of $8 \times 10^{35} \mathrm{~cm}^{-2} \mathrm{~s}^{-1}$, and the experiment is expected to accumulate a data sample of about $50 \mathrm{ab}^{-1}$. With this amount of data, decays sensitive to physics beyond the Standard Model can be studied with unprecedented precision. One promising set of modes are physics processes with missing energy such as $B^{+} \rightarrow \tau^{+} \nu, B \rightarrow D^{(*)} \tau \nu$, and $B \rightarrow K^{(*)} \nu \bar{\nu}$ decays. The $B \rightarrow K^{(*)} \nu \bar{\nu}$ decay provides one of the cleanest experimental probes of the flavour-changing neutral current process $b \rightarrow s \nu \bar{\nu}$, which is sensitive to physics beyond the Standard Model. However, the missing energies of the neutrinos in the final state makes the measurement challenging and requires full reconstruction of the spectator $B$ meson in $e^{+} e^{-} \rightarrow \Upsilon(4 S) \rightarrow B \bar{B}$ events. This report discusses the expected sensitivities of Belle II for these rare decays.
\end{abstract}

Keywords: Missing Energy; Rare Decay; Belle II.

\section{New Physics Searches with Missing Energy at Belle II}

The Belle II detector at the Super-KEKB accelerator will collect data from asymmetric $e^{+} e^{-}$collisions at centre-of-mass energies equivalent to those of $\Upsilon(n S)$ resonances, mainly the $\Upsilon(4 S)$, which is perfectly suited for the studies of $B$ decays. With respect to its predecessor Belle, the Belle II detector will be taking data at an accelerator with a 40 times higher luminosity, thus has to cope with 40 times higher event rates, as well as with backgrounds rates higher by a factor of 10 to 20. In order to maintain the excellent performance, an upgrade of the detector is ongoing. A full discussion can be found in the Belle II Technical Design Report ${ }^{1}$.

Leptonic and semi-leptonic and rare $B$ decays are sensitive to probe new physics (NP) beyond the Standard Model (SM). The challenging is that there is one or even multiple missing neutrinos in the final state which can not be directly detected by

This is an Open Access article published by World Scientific Publishing Company. It is distributed under the terms of the Creative Commons Attribution 4.0 (CC-BY) License. Further distribution of this work is permitted, provided the original work is properly cited. 
Table 1. Tag-side efficiency: number of correctly reconstructed tag-side $B$ mesons divided by the total number of $\Upsilon(4 S)$ events.

\begin{tabular}{cccc}
\hline Tag & FR @ Belle & FEI @ Belle MC & FEI @ Belle II MC \\
\hline Hadronic $B^{+}$ & $0.28 \%$ & $0.49 \%$ & $0.61 \%$ \\
Semileptonic $B^{+}$ & $0.67 \%$ & $1.42 \%$ & $1.45 \%$ \\
Hadronic $B^{0}$ & $0.18 \%$ & $0.33 \%$ & $0.34 \%$ \\
Semileptinic $B^{0}$ & $0.63 \%$ & $1.33 \%$ & $1.25 \%$ \\
\hline
\end{tabular}

the detector. As a unique analysis technique at $B$ factories, the $B$-tagging plays an important role in the measurement of these decays. This technique reconstructs one of the $B$ mesons (tag-side $B$ ) and infers strong constraints for the other $B$ meson (signal-side $B_{\text {sig }}$ ) in the event using the precisely known initial state of the $\Upsilon(4 S)$. The background can be drastically reduced by discarding events with extra tracks or extra energy clusters in the rest of the event. This technique was already employed at Belle, known as Full Reconstruction (FR) and has achieved a great success. Comparing to FR, at Belle II, the further developed Full Event Interpretation (FEI) is more inclusive and provides more automation and analysis-specific optimizations. The basic idea of the FEI is to reconstruct the particles and train the multivariate classifiers (MVC) in a hierarchical approach. At first, the final-state particle candidates are selected and corresponding classification methods are trained using the detector information. Then, intermediate particle candidates are reconstructed and a MVC is trained for each employed decay channel. Finally, the MVC combines all information about a candidate into a single value, the signal-probability. The performance of the FEI is estimated by the number of correctly reconstructed tag-side $B$ mesons divided by the total number of $\Upsilon(4 S)$ events, and the tagging efficiencies are compared to Belle as summarized in Table 1. We see that the FEI has higher tagging efficiencies comparing to FR method.

\section{Search for $B^{+} \rightarrow \tau^{+} \nu$}

In the SM, the tree-level process $B^{+} \rightarrow l^{+} \nu(l=e, \mu, \tau)$ happens via exchanging the $W$ boson, the branching fraction is given by

$$
\mathcal{B}\left(B^{+} \rightarrow l^{+} \nu_{l}\right)_{\mathrm{SM}}=\frac{G_{F}^{2} m_{B} m_{l}^{2}}{8 \pi}\left(1-\frac{m_{l}^{2}}{m_{B}^{2}}\right)^{2} f_{B}^{2}\left|V_{\mathrm{ub}}\right|^{2} \tau_{B},
$$

where $G_{F}$ is the Fermi coupling constant, $V_{\mathrm{ub}}$ is the Cabibbo-Kobayashi-Maskawa (CKM) matrix element, $m_{B}$ and $m_{l}$ are the mass of the $B$ meson and lepton, respectively, $\tau_{B}$ is the lifetime of the $B$ meson and $f_{B}$ is the decay constant. Due to helicity suppression, the branching fraction depends strongly on the mass of the lepton, thus, $B^{+} \rightarrow \tau^{+} \nu$ is expected to have the largest leptonic branching fraction. The NP could significantly enhance or suppress the branching ration via exchanging a charged Higgs boson from supersymmetry or two-Higgs doublet models (2HDM). In the absence of new physics, purely leptonic decays can provide direct 


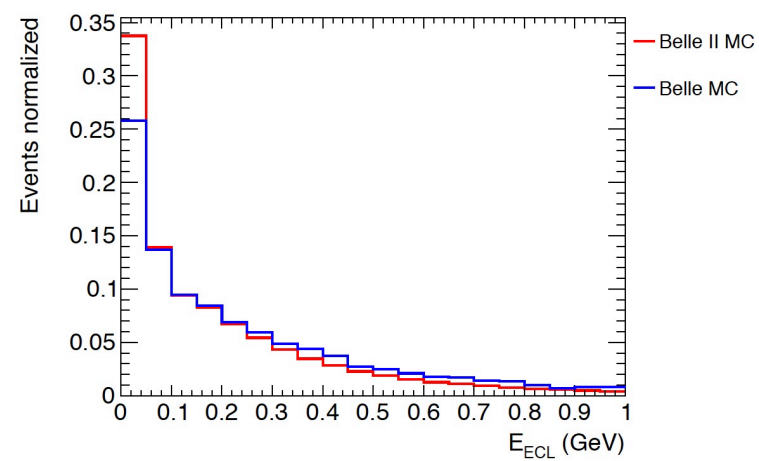

Fig. 1. Comparison of signal $E_{\mathrm{ECL}}$ distribution for $B \rightarrow \tau \nu$ at Belle II MC and the Belle measurement with hadronic tag.

Table 2. Expected uncertainties on the $B \rightarrow \tau \nu$ branching ratio with $50 \mathrm{ab}^{-1}$ data at Belle II.

\begin{tabular}{cccc}
\hline & statistical uncertainty (\%) & systematic uncertainty (\%) & total (\%) \\
\hline hadronic tag & 4.1 & 4.6 & 6.2 \\
semileptonic tag & 2.7 & 4.5 & 5.3 \\
\hline
\end{tabular}

determinations of $\left|V_{\mathrm{ub}}\right|$. This decay has been searched for experimentally, the latest measured branching fraction is $\mathcal{B}\left(B^{+} \rightarrow \tau^{+} \nu\right)=0.72_{-0.25}^{+0.27}$ (stat.) \pm 0.11 (syst.) $\times 10^{-4}$ from hadronic tagging ${ }^{2}$ and $\mathcal{B}\left(B^{+} \rightarrow \tau^{+} \nu\right)=1.25 \pm 0.28$ (stat.) \pm 0.27 (syst.) $\times$ $10^{-4}$ from semileptonic tagging ${ }^{3}$, the combined result is $\mathcal{B}\left(B^{+} \rightarrow \tau^{+} \nu\right)=0.91 \pm$ 0.19 (stat.) \pm 0.11 (syst.) $\times 10^{-4}$ with a significance of $4.6 \sigma$. This result is consistent with the SM expectation but the measurements are still statistically limited. An analysis using the Belle II full simulation has been performed. The Fig. 1 shows the distribution of extra energy deposited in the calorimeter $\left(E_{\mathrm{ECL}}\right)$ of the signal ${ }^{4}$, it indicates that, despite of the increased beam backgrounds, the resolution at Belle II is better than that at Belle. Extrapolating the systematic effects from the most recent Belle analyses and considering the full Belle II statistics, uncertainties of the order of $6 \%$ on the branching fraction are expected for both the hadronic and semileptonic tagging, see Table. 2.

\section{Search for $B \rightarrow D^{(*)} \tau \nu$}

The decays $B \rightarrow D^{(*)} \tau \nu$ are described at the quark level as $b \rightarrow c \tau \nu$ tree-level transitions that proceed in the SM through the exchange of a virtual $W$ boson. In the SM, the only differences between $B \rightarrow D^{(*)} \tau \nu$ and $B \rightarrow D^{(*)} \mu \nu$ is the mass of the lepton. Typically, the lepton universality is tested using the ratio

$$
R\left(D^{(*)}\right)=\frac{\mathcal{B}\left(B \rightarrow D^{(*)} \tau \nu_{\tau}\right)}{\mathcal{B}\left(B \rightarrow D^{(*)} l \nu_{l}\right)}(l=e, \mu)
$$


because of large reductions of theoretical uncertainties in form factors and $V_{\mathrm{cb}}$. The ratio is sensitive to the additional amplitudes, for instance, new physics involving an intermediate charged Higgs boson or leptoquarks model. This value has been measured by the BaBar, Belle and the LHCb collaborations. The current world average is about $4.1 \sigma$ away from the SM predication. Therefore, measurements of $B \rightarrow D^{(*)} \tau \nu$ are considered of great importance at Belle II. In addition to $R\left(D^{(*)}\right)$, the NP could also affect the polarization of $\tau$ lepton, which is defined by $P_{\tau}\left(D^{*}\right)=$ $\frac{\Gamma^{+}-\Gamma^{-}}{\Gamma^{+}+\Gamma^{-}}$, where the $\Gamma^{+}$is the decay rate with the $\tau$ helicity $+1 / 2$ and $\Gamma^{-}$the $\tau$ helicity $-1 / 2$. The $\tau$ polarization has been measured at Belle recently ${ }^{5}$. Based on the existing results from Belle and expected improvements at Belle II, the precisions in the $R_{D^{(*)}}$ and $P_{\tau}\left(D^{*}\right)$ are estimated ${ }^{4}$. The projects of $R_{D}$ vs $R_{D^{*}}$ plane at Belle II are shown and compared to the current results and the SM predictions in the left plot of Fig. 2. The projects of $R_{D^{*}}$ vs $P_{\tau}\left(D^{*}\right)$ plane are shown in the right plot of Fig. 2. The expected precision on $R_{D^{(*)}}$ and $P_{\tau}\left(D^{*}\right)$ at Belle II is summarized in Table. 3. Furthermore, comparing to the two-body decay of $B \rightarrow \tau \nu$, the process $B \rightarrow D^{(*)} \tau \nu$ feature a richer phenomenology. It will be instructive to study on the differential branching fraction depending on the momentum transfer to the lepton pair, $q^{2}=\left(p_{\tau}+p_{\nu}\right)^{2}=\left(p_{B}-p_{D^{(*)}}\right)^{2}$, which is essential to distinguish NP.
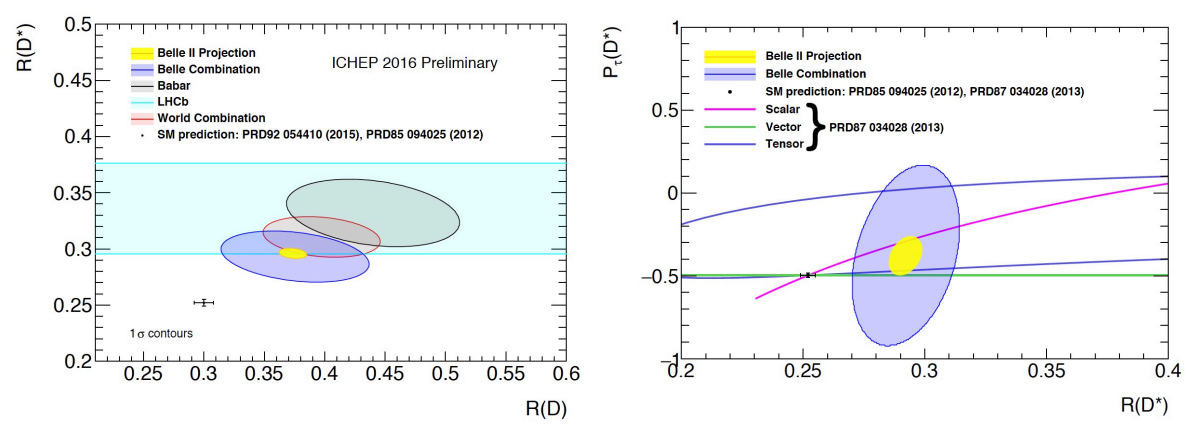

Fig. 2. Expected Belle II constraints on the $R_{D}$ vs $R_{D^{*}}$ plane (left) and the $R_{D^{*}}$ vs $P_{\tau}\left(D^{*}\right)$ plane (right) compared to the existing experimental constraints from Belle. The SM predictions are also indicated.

Table 3. The expected precision on $R_{D^{(*)}}$ and $P_{\tau}\left(D^{*}\right)$ at Belle II. The first and the second values are the expected statistical and systematic uncertainties, shown as the relative (absolute) values for $R_{D^{(*)}}$ $\left(P_{\tau}\left(D^{*}\right)\right)$.

\begin{tabular}{|c|c|c|c|c|c|}
\hline & $50 \mathrm{ab}^{-1}$ & 1 & $50 a b^{-1}$ & & $50 \mathrm{ab}^{-1}$ \\
\hline$R_{D}$ & $(2.0 \pm 2.5) \%$ & $\mid R_{D^{*}}$ & $(2.0 \pm 2.5) \%$ & $P_{\tau}\left(D^{*}\right)$ & $0.06 \pm 0.04$ \\
\hline
\end{tabular}




\section{Search for $B \rightarrow K^{(*)} \nu \bar{\nu}$}

Flavor-changing neutral-current (FCNC) process is prohibited in SM at tree-level, it can only occur via one-loop box or electroweak penguin diagrams. The $B \rightarrow K^{(*)} \nu \bar{\nu}$ is theoretically clean because of the absence of long-distance hadronic effects, thus is the golden mode at Belle II. Due to the exact factorization, the precision of the SM prediction for the branching ratios of $B \rightarrow K^{(*)} \nu \bar{\nu}$ is mainly limited by the $B \rightarrow K^{(*)}$ form factors and by the knowledge of the relevant CKM elements. Experimental searches have been performed with the BaBar and Belle data, no evidence for signal has been found. An analysis on Belle II full simulation has been performed. In order to evaluate the impact of machine background on $B \rightarrow$ $K^{(*)} \nu \bar{\nu}$ searches, the generic MC samples superimposed to the nominal machine background are studied. The quantity $E_{\text {miss }}^{*}+c p_{\text {miss }}^{*}$ is much less correlated to the $\nu \bar{\nu}$ invariant mass than $E_{\text {miss }}^{*}$ or $p_{\text {miss }}^{*}$ alone, making it suitable for a modelindependent analysis. The Fig. 3 shows the $E_{\text {miss }}^{*}+c p_{\text {miss }}^{*}$ distributions for the $K^{*+} \rightarrow K^{+} \pi^{0}$ channel for signal and generic MC samples. From the study, we see that the detector performances and the reconstruction algorithms are robust against machine background. The Belle II sensitivity projection is based on recent Belle measurement with the hadron tag sample and considering the improvement factors ${ }^{6}$. The signal yields is estimated from the SM branching fractions ${ }^{7}$, the projects are summarized in Table. 4 . It is expected that $B \rightarrow K^{+} \nu \bar{\nu}$ and $B \rightarrow K^{* 0} \nu \bar{\nu}$ will be observed with about $18 \mathrm{ab}^{-1}$ data and with $50 \mathrm{ab}^{-1}$, the sensitivities of the branching fraction will be $12 \%$ and $11 \%$, respectively. Once the $K^{*}$ modes are observed, measurements of differential branching fraction and $K^{*}$ polarization will be important subjects.

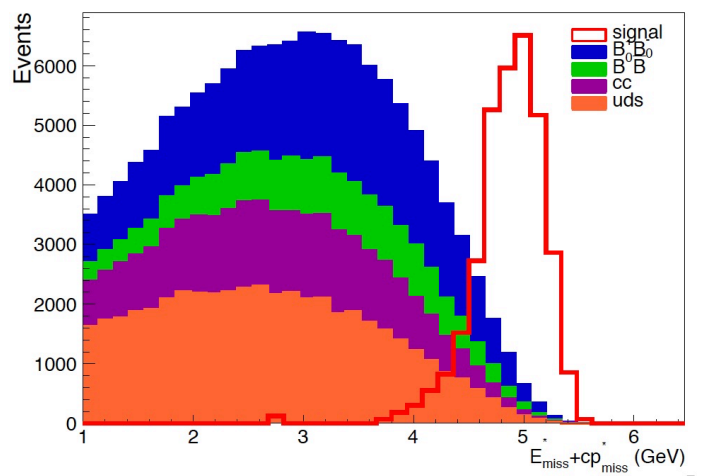

Fig. 3. Distribution of $E_{\text {miss }}^{*}+c p_{\text {miss }}^{*}$ for the signal and the generic MC samples, for the $K^{*+} \rightarrow$ $K^{+} \pi^{0}$ channels with nominal background after all the selection criteria applied but the ones on $E_{\text {miss }}^{*}+c p_{\text {miss }}^{*}$ and $E_{\mathrm{ECL}}$. The number of generic MC events corresponding to an integrated luminosity of $1 \mathrm{ab}^{-1}$ while the signal normalization is arbitrary. 
Y. Guan

Table 4. Projections for the statistical uncertainties on the $B \rightarrow K^{(*)} \nu \bar{\nu}$ branching fractions.

\begin{tabular}{|c|c|c|c|c|c|c|c|c|}
\hline Mode & $\mathcal{B}\left[10^{-6}\right]$ & $\begin{array}{c}\text { Efficiency } \\
\text { Belle } \\
{\left[10^{-4}\right]}\end{array}$ & $\begin{array}{c}N_{\text {Backg. }} \\
711 \mathrm{fb}^{-1} \\
\text { Belle }\end{array}$ & $\begin{array}{c}N_{\text {sig-exp. }} \\
711 \mathrm{fb}^{-1} \\
\text { Belle }\end{array}$ & $\begin{array}{l}N_{\text {Backg. }} \\
50 \mathrm{ab}^{-1} \\
\text { Belle II }\end{array}$ & $\begin{array}{c}N_{\text {sig-exp. }} \\
50 \mathrm{ab}^{-1} \\
\text { Belle II }\end{array}$ & $\begin{array}{c}\text { Statistical } \\
\text { Error } \\
50 \mathrm{ab}^{-1}\end{array}$ & Total \\
\hline$B^{+} \rightarrow K^{+} \nu \bar{\nu}$ & 4.68 & 5.68 & 21 & 3.5 & 2960 & 245 & $20 \%$ & $22 \%$ \\
\hline$B^{0} \rightarrow K_{S}^{0} \nu \bar{\nu}$ & 2.17 & 0.84 & 4 & 0.24 & 560 & 22 & $94 \%$ & $94 \%$ \\
\hline$B^{+} \rightarrow K^{*+} \nu \bar{\nu}$ & 10.22 & 1.47 & 7 & 2.2 & 985 & 158 & $21 \%$ & $22 \%$ \\
\hline$B^{+} \rightarrow K^{* 0} \nu \bar{\nu}$ & 9.48 & 1.44 & 5 & 2.0 & 704 & 143 & $20 \%$ & $22 \%$ \\
\hline \multicolumn{9}{|l|}{$B^{+} \rightarrow K^{*} \nu \bar{\nu}$} \\
\hline combined & & & & & & & $15 \%$ & $17 \%$ \\
\hline
\end{tabular}

\section{Summary}

Belle II will be a unique environment to study $B$ decays with missing energy, which is very sensitive to indirect new physics effects. Belle II will have a factor of 40 in luminosity with respect to the first generation $B$-factories, will have the capability to perform studies of $B$ mesons decays with unprecedented precision. Previously observed anomalies, such those observed in $B \rightarrow D^{(*)} \tau \nu$ can be resolved with a few $\mathrm{ab}^{-1}$ of data, while very rare decays $B \rightarrow K^{(*)} \nu \bar{\nu}$ can be probed at about $5 \sigma$ level with the full Belle II data. Full simulation studies, with the current knowledge of the machine background rates, have proven the detector performance and the reconstruction algorithms to be robust against machine background. The first data taking runs for physics analyses at Belle II are anticipated to begin in 2018.

\section{References}

1. T. Abe et. al, Belle II Collaboration, arXiv:1011.0352 (2010).

2. I. Adachi et. al, Belle Collaboration, Phys. Rev. Lett. 110, 131801 (2013).

3. B. Kronenbitter et. al, Belle Collaboration, Phys. Rev. D92, 051102 (2015).

4. E. Kou, P. Urquijo, the Belle II collaboration and the B2TiP theory community, The Belle II Physics Book, to appear in Prog. Theor. Exp. Phys..

5. S. Hirose et. al, Belle Collaboration, Phys. Rev. Lett. 118, 211801 (2017).

6. P. Urquijo, BELLE2-MEMO-2016-008.

7. D. M. Straub, BELLE2-MEMO-2016-007. 